\title{
LA EXPRESIÓN CORPORAL COMO FORMA DE COMUNICACIÓN ESENCIAL
}

\author{
S. CARNERO-SIERRA ${ }^{1}$, A. M. P. AMÉZAGA ${ }^{2}$ \\ Universidad de Oviedo ${ }^{1}$ \\ Escuela Superior de Arte Dramático del Principado de Asturias ${ }^{2}$ \\ carnerosusana@uniovi.es ${ }^{1}$ \\ Artigo submetido em 03/04/2019 e aceito em 23/06/2019 \\ DOI: $10.15628 /$ holos.2019.8477
}

\section{RESUMEN}

La disciplina denominada Expresión Corporal comprende un campo multidisciplinar en el que confluye el terreno educativo y comunicativo, pero también el artístico y terapéutico. El acto de comunicar con el cuerpo supone un hecho indesligable en la ontogenia del individuo, hecho tradicionalmente estudiado en el ámbito académico por el campo de la comunicación no verbal. En este trabajo se realiza una propuesta para reformular el enfoque de investigación de la también denominada Técnica y Expresión de Movimiento, definiendo los orígenes artísticos de la misma y situándola como disciplina de características independientes respecto de la danza y la comunicación no verbal. De esta forma, se contemplan nuevas posibilidades en su estudio, conectando su origen artístico con sus potencialidades en el terreno comunicativo, educativo y socio-terapéutico.

PALABRAS-CLAVE: Comunicación no verbal, expresión corporal, semiología, lenguaje simbólico, educación artística.

\section{BODILY EXPRESSION AS ESSENTIAL COMMUNICATIVE WAY}

\begin{abstract}
Bodily Expression is a multidisciplinary field in which educational, communication, artistic and therapeutic matters come together. The action of corporal communication means an inseparable fact in individual ontogeny of subject, usually studied by non-verbal communication field in academic circles. This work presents a proposal for reformulation of research focus
\end{abstract}

in Technique and Expression of Movement. In the following, it will be stated the artistic origins of this field, framing its characteristics independently from dance or non-verbal-communication. This way, new possibilities are contemplated, connecting artistic origin of bodily expression with its potential in communication, educational and socio-therapeutically fields.

KEYWORDS: Non-verbal communication, movement education, semiotics, symbolic language, artistic education. 


\section{PRESENTACIÓN}

La disciplina tradicionalmente denominada Expresión Corporal (EC) es tan amplia y variada como los ámbitos en los que la misma se ve incluida. Es así que la Expresión Corporal se puede entender como un lenguaje no codificado, entendiéndose por codificación la especificación sistemática de reglas y signos que sirve para emitir y entender un mensaje. Los especialistas en semiología coinciden en que este sistema de signos y reglas no tiene por qué ser solo de naturaleza lingüística, sino también gestual o incluso musical. Por ejemplo, desde el contexto teatral, un código es la convención por la que se asocian unos determinados contenidos con elementos concretos dentro de un sistema expresivo.

Considerando el teatro como el lugar donde se explora e investiga sobre el cuerpo en movimiento, es decir, el centro de estudio del cuerpo expresivo teatral, es acertado pensar que el traslado de este conocimiento a otros espacios como el educativo, comunicativo y el terapéutico ha enriquecido la disciplina de la EC ofreciendo importantes aportaciones. Desde el campo educativo y artístico, entornos donde se ha desarrollado históricamente la también denominada Técnica y Expresión de Movimiento (Schinca, 2010), la Expresión Corporal es el ámbito de conocimiento donde la gramática del cuerpo y sus factores de movimiento son la base para para construir una forma de comunicación independiente.

Tradicionalmente se han desarrollado dos caminos principales por los que explorar este lenguaje del cuerpo. Entendido únicamente como comunicación no verbal, tiene su referencia en el pensamiento de Knapp (Knapp, Wiemann \& Daly, 1978) y Flora Davis (1998). Ambos autores destacaron la importancia del lenguaje corporal y la comprensión del mismo, describiendo el comportamiento no verbal como el acento que aporta un mayor significado al mensaje oral. En este sentido, también se encuentra el trabajo de Ekman (Ekman \& Friesen, 1969) el cual ha generado un importante desarrollo en la investigación de la expresión de las emociones desde la psicología experimental de la micro gestualidad y la detección de contradicciones entre la comunicación verbal y no-verbal. Sin embargo, el devenir más fructífero de la Expresión Corporal ha venido de la mano de las disciplinas educativas y artísticas. En el campo educativo, no es raro encontrar amplios desarrollos que no resultan extraños a la investigación en comunicación. Recientemente, autoras alejadas del entorno escénico, pero más cercanas al campo educativo de lo físico, señalan que desde la EC se entiende que el cuerpo tiene la posibilidad de ser un instrumento con el que expresarse a través de un lenguaje propio (García, Pérez \& Calvo, 2003).

El desarrollo de la también llamada Técnica de Movimiento Expresivo ha concurrido además un camino diferente, siendo el campo artístico el que más prácticos y variados marcos ha explorado, haciendo de la EC una disciplina que permite comunicar emociones o situaciones de manera significativa e intensa. Por ejemplo, dos de los paradigmáticos ejemplos artísticos del uso del movimiento como acto comunicativo eminentemente no verbal, se encuentran en el arte de la Mima de Étienne Decroux (Angotti \& Herr, 1974) y en corrientes desde la danza expresiva próximas a estudiosos del movimiento como Rudolph Von Laban (Laban, 1960). Sin embargo, la Expresión Corporal posee una identidad propia que la diferencia de estas otras artes, precisamente por su énfasis explícito en el proceso comunicativo. El hecho de que los factores del movimiento que se 
utilizan en la danza, en el mimo y en la EC sean los mismos, así como considerar la influencia de precursores y autores comunes en las tres disciplinas ha conducido a generar confusión en la especificidad de cada uno de estos campos. Es común el objetivo perseguido, la expresión de emociones, así como el lugar en donde realizar las creaciones, es decir, el espacio escénico en donde hacer y representar ante un público. Entonces, ¿cuál es la diferencia entre estas disciplinas?, ¿qué es lo que ofrece la EC distinto al resto de otras artes del movimiento?

Es por este motivo que los siguientes epígrafes ofrecen un panorama de los orígenes artísticos de la Expresión Corporal, exponiéndose a continuación las características que la hacen una disciplina comunicativa independiente. Se recorrerá una perspectiva que, desde el arte y la educación actoral, conduce a la EC a ser estudiada como una forma de comunicación esencial, que posee una gramática propia y un objetivo comunicativo sintético. Finalmente se expondrán las conclusiones por las que, el estudio de la EC abre nuevas vías de investigación e intervención para la comunicación no verbal en terrenos sociales y científicos, más allá de lo escénico.

\section{LOS ORÍGENES ESCÉNICOS DE LO CORPORAL COMO FORMA DE COMUNICACIÓN Y EXPRESIÓN.}

El trabajo de toma de conciencia del propio cuerpo y el estudio de los factores en los que se puede descomponer todo movimiento, se hacen imprescindibles al subrayar la importancia de la expresión no verbal en la comunicación cotidiana. Esto es así porque la complejidad del dominio corporal y la escucha propioceptiva, así como la exigencia en la calidad de movimiento significativo, hacen de esta materia un arte que trabaja con la expresión de emociones e incluso de ideas abstractas. A veces estas emociones se muestran tan complejas que la no intervención de la palabra se hace necesaria. Junto a esta necesidad aparece el gesto (ver Figura 1), con el que la persona es capaz de expresar sus sentimientos con profundidad, sin intermediario. Es por esto que el estudio de la situación de máximo desempeño de esta tarea, es decir la educación del actor/actriz y la tradición teatral del uso del cuerpo en escena, genera interesantes puntos de vista en el estudio de la comunicación a través de las formas corporales.

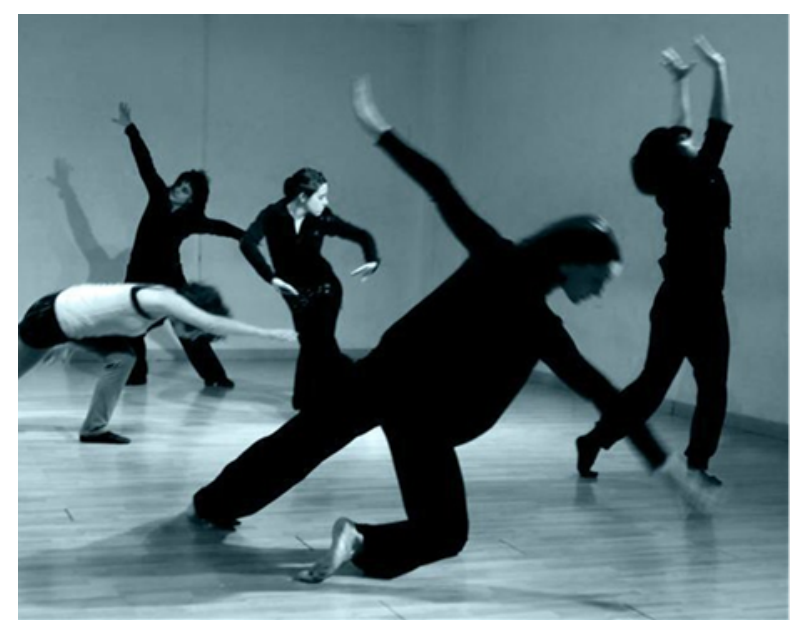

Figura 1. Extracto de una sesión de Expresión Corporal según el método Schinca: ejemplos de gesto significativo donde se observan algunos de los factores de movimiento como los niveles, el trabajo en planos y el contramovimiento. 
En relación a esta forma de expresar hay que resaltar que, para Artaud el objetivo del teatro sería expresar objetivamente a través de la gestualidad del actor/actriz (Finter \& Griffin, 1997). Trabajar en esta dimensión supone saber utilizar las herramientas corporales, comenzando por la toma de consciencia de la gama de posibilidades que ofrece el cuerpo, así como saber utilizar el movimiento en el espacio y el tiempo. Esta figura referente en la historia del teatro considera que "el actor es un atleta del corazón" (Artaud, 1986, p.147). Un atleta que no prepara su cuerpo para competir y ganar, sino para transformarse en tantas figuras, formas y diseños corporales como dictaminen sus personajes.

A finales del siglo XIX, la necesidad de cubrir las carencias de la enseñanza llevó a los maestros de teatro a experimentar con nuevos métodos de educación teatral. Para Aslan (1979), una de las aportaciones fundamentales de todas estas escuelas fue la importancia que dieron a la formación corporal sistemática del actor. Dentro de estas primeras escuelas, apelar en primer lugar al director y actor teatral ruso Stanislavski (1863-1938) para señalar un cambio significativo en cuanto a los métodos de interpretación, es un lugar común (Barba \& Savarese, 1988; Ruiz Osante, 2008) que no podemos olvidar para entender el papel de la EC en la formación actoral, pues revolucionó el arte escénico al redefinir la misión del actor.

En el Vieux-Colombier de Copeau (1879-1949) las prácticas deportivas como la natación, la esgrima o la acrobacia, utilizadas para la preparación del cuerpo del actor/actriz, se vieron rápidamente acompañadas de la música y en concreto, de la vivencia del ritmo que tenía como base las enseñanzas de Dalcroze. En referencia a este trabajo corporal en el ámbito de la formación del actor/actriz, Cohen (2013) atribuye a Copeau la utilización del término Expresión Corporal. Por otro lado, Jan Doat, en su obra "La expresión corporal del comediante" (Doat, 1976), reconoce la influencia de Charles Dullin (1885-1949) en su concepto de Expresión Corporal, lo cual corrobora la importancia que la escuela francesa dio y sigue dando, al control y dominio del cuerpo, a su expresividad y a la elocuencia corporal.

Igualmente, la biomecánica de Meyerhold (1874-1940) fue un reflejo del valor que este le daba al aspecto expresivo del cuerpo. La preparación corporal del actor/actriz tenía un fin, el cuerpo debía volverse altamente expresivo en el espacio que le rodeaba (Whitehead, 2017). En él, el actor en movimiento diseña de forma precisa la forma, para poder expresar una emoción sin necesidad de vivirla (Barba, 2003). A continuación, en este periodo se inserta el trabajo del Mimo Corpóreo de Decroux (Lust, 1971), el cual entiende que los movimientos del cuerpo evocan los del alma. Creó un estilo de actuación con el que poder expresar aquello que la palabra no puede decir, aunque la palabra nunca quedó excluida en el programa de desarrollo del Mimo. Este arte cuyo origen se remonta a Grecia y Roma clásicas y se desarrolla con la pantomima blanca del siglo XIX, es desarrollado por Decroux con máxima codificación para, a través del estudio de los ejes en el espacio, los ejes corporales, las direcciones, los triples dibujos y en general el análisis del movimiento, crear una gramática corporal. Los ejercicios que plantea Decroux a sus alumnos son secuencias de movimientos en los que se da una forma física que se repite y se interioriza con la repetición. Los ejercicios son como un léxico o glosario que el alumno aprende y luego utiliza en la creación, comunicando desde la forma a través de una técnica precisa y virtuosa. En este punto se contempla con claridad el hecho de que también el movimiento corporal puede trabajar con una gramática. 
En esta línea, cabe destacar de nuevo el trabajo reflexivo de Artaud sobre la importancia de la expresión objetiva del gesto. El gesto y en general todo lenguaje que se transmite en el espacio, puede alcanzar con mayor precisión aquellas actitudes a las que no llega la palabra. Para este autor dar más importancia al lenguaje hablado o a la expresión verbal que a la expresión objetiva de los gestos y todo lo que afecta al espíritu por medio de elementos sensibles en el espacio, es volver la espalda a las necesidades físicas de la escena y destruir sus posibilidades (Artaud, 1986).

La exactitud del movimiento y la precisión del gesto en el espacio es para Lecoq el objetivo a alcanzar y para ello, el cuerpo del actor debe comprometerse con el trabajo, relacionando la emoción directamente con el movimiento (Lecoq, 2003). Por otra parte, el trabajo de Grotowski (1933-1999), tuvo como materia de investigación el entrenamiento actoral, de ahí el término laboratorio, en donde el actor/actriz experimentaba con la intensa relación entre el mundo interior personal y su manifestación externa a través del movimiento y del cuerpo en la acción (Grotowski, Wiewiorowski \& Morris, 1967). Para ello creó un entrenamiento corporal específico, con el cual se fueron desarrollando una serie de ejercicios, algunos de ellos de alta precisión técnica, que con el tiempo quedaron consolidados dentro del método Grotowskiano, así como el concepto de teatro laboratorio, donde el actor/actriz se centraba en el trabajo evolutivo de su propio cuerpo como generador de la dramaturgia y la interpretación (Laster, 2012).

Más cercanos en el tiempo, Barba, Oida, Littelwood y Bogart siguen con la tradición establecida por los autores surgidos en el Vieux-Colombier, en cuanto a la integración del cuerpo en la formación actoral. Así, Barba guía al actor hacia el encuentro del cuerpo fuera de lo cotidiano y vivo en la acción, con el que poder liberarse de los automatismos de los hábitos y alejarse de representar siempre un cuerpo humano parecido a sí mismo (Barba \& Savarese, 1988; Watson, 2013). El trabajo corporal en Oida es mucho más que entrenar, calentar, fortalecer o flexibilizar el cuerpo. Este autor plantea que la preparación corporal posibilita que el actor/actriz comprenda desde su cuerpo, la propia acción de actuar. Percibir desde el cuerpo antes que teorizar para aprender (Oida, 1993). Joan Littelwood (1914-2002), en colaboración con Jean Newlove, alumna y compañera profesional de Laban, recoge las investigaciones de este último sobre el movimiento humano, adaptando la idea de esfuerzo a la caracterización física y vocal de los personajes, buscando la economía de acciones, la precisión del movimiento y el manejo del ritmo en la creación de las partituras de los espectáculos (Ruiz Osante, 2008).

Estos autores, directores o actores utilizaron y revelaron la importancia del cuerpo en movimiento como base de la comunicación teatral. A partir de ellos los términos training actoral o entrenamiento físico, fueron adquiriendo importancia y se establecieron como parte indivisible de la formación escénica. Muy relacionados con estas escuelas y maestros teatrales, compartiendo muchas veces escenario pedagógico con ellos, aparecen otros creadores que establecen la raíz y principios básicos de la Expresión Corporal como disciplina independiente (Ferrari, 2015). Estas personalidades corresponden a Delsarte, Dalcroze, Laban y Bode, de los que a continuación señalaremos sus aportaciones más significativas. Conocer los aspectos más relevantes de su obra, supone no solo un esfuerzo de organización histórica desde el arte teatral, sino también un esfuerzo por identificar los conceptos comunes que asimismo se observan en interacciones básicas de la comunicación humana. 


\section{PRINCIPIOS BÁSICOS DE LA EXPRESIÓN CORPORAL.}

François Delsarte (1811-1871) fue un músico, cantante, actor y pedagogo en el ámbito del arte dramático. Delsarte se entregó de lleno a la investigación de la correspondencia entre los sentimientos y sus manifestaciones expresivas corporales y vocales. Sin haber creado un sistema o método, Delsarte desarrolla los principios que hoy en día se consideran la base de la Expresión Corporal. Su denominado Principio de Correspondencia, consiste en entender que cada función del cuerpo posee su correspondencia en una función espiritual y viceversa (Odom, 2005). Esta relación intrínseca fue en su época un verdadero descubrimiento, crucial para la educación integral a través del trabajo corporal. A esta relación se añade el Principio de Oposición, por el que todo segmento corporal en movimiento tiene su movimiento opuesto, necesario en el establecimiento del equilibrio. Por último, se encuentra el Principio de Sucesión definido por el recorrido necesario del movimiento a través de las distintas articulaciones, las cuales reaccionan al tono muscular y la activación de cada articulación inmediatamente anterior.

A partir de Delsarte, hubo otras aportaciones como las de Dalcroze (1865-1950), compositor y educador, creador del método de enseñanza musical denominado Rítmica o método Dalcroze (Odom, 2005). Esta metodología tiene como objetivo crear, con la ayuda del ritmo, una corriente de comunicación rápida y regular entre la fisiología y la persona (Seitz, 2005). Su sistema de enseñanza de la música a través del movimiento y los matices dinámicos, agógicos y expresivos influyó y aún influye, en la educación por el movimiento.

Es destacable recordar que los matices agógicos se refieren a la duración de las figuras musicales o, en este caso, del movimiento. En cambio, los dinámicos hacen referencia a los matices de intensidad. El sonido, al igual que el movimiento, se produce dentro de una gama muy amplia de intensidades, las cuales abarcan desde un fortísimo (fff) a un pianissimo (ppp). En el caso de la música los sonidos aumentan o disminuyen de volumen con la intensidad. En la Expresión Corporal, los cuerpos actúan con mayor o menor fuerza muscular dependiendo de la intensidad del movimiento. El grado de tensión muscular con que se desarrolla el movimiento (Sainsbury, 1964) también origina distintos ritmos, que requieren de un cierto control y dominio del cuerpo para manejar las distintas gamas de intensidades en distintas zonas expresivas corporales.

En música, el ritmo se define como la ordenación de sonidos cortos, largos y sus silencios, siendo el tempo la velocidad de ejecución de esa ordenación. En el campo de la Expresión Corporal también es factible esta definición, sustituyendo la materia prima de la música, el sonido, por la materia prima de la Expresión Corporal: el cuerpo y sus movimientos y silencios. El ritmo es un elemento vital (Kotz, Ravignani, \& Fitch, 2018) que surge de la ordenación y combinación de las duraciones del movimiento, de su agógica y de la acentuación del mismo en la creación de distintas dinámicas. De la misma manera, los silencios y sus distintas duraciones forman parte del lenguaje corporal, desarrollándose así la capacidad de escucha y concentración para la respuesta y reacción hacia el otro/a o los otros/as.

Por otro lado, especialmente valiosa ha sido, en el campo del análisis del movimiento humano, la aportación de Rudolf von Laban (1879-1958), creando las bases del análisis del 
movimiento humano, sintetizando una disciplina denominada Movimiento Creativo o Danza Libre, como se le llamó en ese tiempo. Sus investigaciones se centran en las leyes generales del movimiento, creando de este modo una teoría básica. Así como las técnicas específicas de danza seleccionan su forma particular de movimiento en función de un estilo, Laban denominó su técnica Danza Libre por ser una técnica liberada de un estilo altamente estereotipado y codificado. Con su estudio y análisis, extrajo lo universal del arte del movimiento en diferentes contextos, incluso en el ámbito del trabajo y las diferentes profesiones. Para ello ahondó en los factores del movimiento, grados de intensidad, relación con el peso, tiempo y espacio que, según sus diferentes combinaciones, otorgan las cualidades expresivas a particulares formas de moverse (Laban, 1960). Las investigaciones de Laban se centran en las leyes generales del movimiento y sus estudios inciden en puntos de vista que se acercan a los esfuerzos cotidianos del ser humano, acercándose incluso al terreno de lo laboral. Es de destacar que algunas de las aportaciones de Laban, encontraron cabida en el contexto del modelo taylorista de trabajo (Rothe, 2012), mostrando que la aplicación del análisis del movimiento de Laban no sólo se limita al campo artístico, sino a la optimización del esfuerzo en cualquier aspecto del movimiento humano. Laban crea de este modo, una teoría del movimiento básica (Laban, 1960), que aclara la importancia del análisis del movimiento a través de los factores que lo componen. Esta teoría es aplicable a todos los campos y su influencia llega hasta nuestros días: se observa en la educación del profesor, el actor y el bailarín, pero también se aplica a todos contextos sociales. Es el caso de las investigaciones realizadas por Abe (2017) en las que se aplica este tipo de análisis al estudio del comportamiento de los pasajeros de metro en hora punta, encontrando patrones recurrentes de movimiento para esta situación social tan concreta.

Por otra parte, hay que destacar que en las primeras décadas del siglo XX diferentes técnicas corporales se empiezan a desarrollar autónomamente, ya sea con fines artísticos, terapéuticos o de conocimiento y progreso personal. Estas técnicas también aportan conocimientos y formas de hacer relevantes para la Expresión Corporal que hoy se conoce. Rudolf Bode (1881-1971) fue el creador de la Gimnasia Expresiva y fundador de la escuela de gimnasia rítmica de Múnich. El legado de Bode a la Expresión Corporal es importantísimo, dado que es quien formula las bases del movimiento natural denominado más comúnmente como movimiento orgánico (Bode, 2014). Renueva los principios pedagógicos en la enseñanza del movimiento y forma a profesores. Aunque los objetivos de las diversas técnicas que integró fuesen diferentes, todas ellas se dirigían sobre la función comunicativa del cuerpo y el movimiento, como una capacidad generadora de un lenguaje, idóneo para trasmitir emociones a otros o, básicamente, de conocimiento de uno mismo.

En síntesis, a partir de estas primeras décadas del siglo XX se puede apreciar cómo, al mismo tiempo, se distinguen dos caminos en el trabajo corporal. Una línea de trabajo que parte de las escuelas europeas que continúan el legado más teatral de Meyerhold (Rusia), Grotowsky (Polonia), Barba (Italia y Noruega), Decroux (Francia), Leqoc (Francia) y Chéjov (Inglaterra) entre otros; y que, como ya se ha mencionado, acuñan el término de entrenamiento, formación física del actor o training actoral. La segunda perspectiva es iniciada con Laban y Dalcroze en Alemania e Inglaterra y que se traslada a América del Sur a través de dos de sus alumnas, Patricia Stokoe e Ingeborg Bayerthald (1905-1986) respectivamente. Stokoe (García et al., 2013) es autora de la Expresión 
Corporal-danza en Argentina y la creadora de un método orientado al desarrollo de la sensibilización corporal. Por otra parte, Bayerthald recala en Uruguay en los años 40, transmitiendo un conocimiento del cuerpo en movimiento que posteriormente trabajaría Schinca, recopilando la tradición en su método de EC. El método Schinca tiene como principio fundamental precisamente considerar la Expresión Corporal como una disciplina independiente del llamado "training del actor" o "trabajo físico del actor" (Ferrari, 2015). Son tres las personas que conciben su trabajo en la denominada EC: Ana Pelegrín, Patricia Stokoe y Marta Schinca. Estas tres pedagogas acuñaron y defendieron el término en elaborados métodos de trabajo, utilizándolo y difundiendo la disciplina de la Expresión Corporal en ámbitos diferentes, en la Educación Física, en la Danza y en el Arte Dramático respectivamente.

\subsection{La Expresión Corporal como disciplina específica e independiente.}

La línea de trabajo que siguen estas tres autoras, aunque como ya se ha explicado tiene unos objetivos comunes al concepto de training actoral, establece una diferencia fundamental con el mismo: la convicción profunda de que la preparación corporal no consiste en una serie de ejercicios, de una determinada dificultad, que exijan al futuro actor/actriz un esfuerzo fundamentalmente físico. Por el contrario, la relación psicofísica es la que debe predominar durante toda la formación y a ser posible, desde el descubrimiento guiado del propio cuerpo en movimiento. Este descubrimiento exige una reflexión personal, bien sea en grupo o individualmente, sobre los procesos de trabajo que expresen la adquisición del conocimiento sobre el cuerpo en movimiento. Indudablemente los métodos de training actoral no excluyen la conexión con la psicología, recurriendo constantemente a la fusión de los procesos personales y los diseños corporales que realiza el actor/actriz. Sin embargo, la diferencia se puede establecer en que, en el entrenamiento actoral, el maestro o profesor es el que crea una secuencia de movimientos, limitando las formas que el cuerpo debe realizar. A partir de ahí y mediante la repetición de estos movimientos, el alumno/a los interioriza y los va nutriendo con la carga personal que surge durante el trabajo. Es decir, en las sesiones de training los futuros actores/actrices imitan los diseños corporales preconcebidos por sus maestros y los repiten hasta lograr una conexión psicofísica.

Por otro lado, la línea de trabajo que establece el estudio y trabajo de la Expresión Corporal se centra en el encuentro de un lenguaje corporal propio desde un armazón de variables corporales por descubrir (Schinca, 2010). La relación psicofísica queda establecida desde el primer momento y la imitación de unas formas instauradas no tienen cabida, ni se conciben como entrenamiento. Este lenguaje personal es quizá lo que caracteriza y diferencia también a la EC de otras artes del movimiento y lo que permite la viabilidad de este trabajo en todo tipo de ámbitos educativos y sociales, incluyendo por completo el campo de la diversidad funcional.

Los objetivos del trabajo de la Expresión Corporal desde la tradición actoral, son diseñados inicialmente para que el actor/actriz pueda utilizar su cuerpo como un auténtico instrumento de transmisión de emociones de las distintas situaciones que se plantee y de las diferentes atmósferas que cree. Objetivos que, por otra parte, son perfectamente aplicables a otros colectivos y planos de la comunicación humana. Para ello, actores de los diferentes escenarios sociales y espectaculares, requieren una percepción completa, una gran conciencia corporal y un estudiado control del cuerpo, con el cual el individuo encuentre su propio lenguaje expresivo personal. Por 
esta razón, la relación del sujeto con su cuerpo no debe ser solamente a través del aprendizaje de movimientos codificados y preestablecidos. Por el contrario, debería primar una perspectiva de autoconocimiento, la cual permita que las distintas experiencias corporales trasformen el cuerpo en la materia prima con la que trabajar, considerando la EC como un vehículo expresivo.

A las particularidades de la EC, hay que sumar el uso de los factores que convierten el movimiento en gesto expresivo. Por ejemplo, es significativo que en cuanto a los métodos empleados para trabajar la EC, Schinca (2010) propone un método en el que se trabaja un vocabulario nacido de la propiocepción corporal, los cambios de intensidad tónica muscular y la percepción del espacio y el tiempo, como reguladores concretos de la expresión del cuerpo. Los diseños corporales resultantes de modificar las variables del movimiento fisiológico, espaciales y temporales repercuten en los tres principios fundamentales del movimiento formulados por Delsarte: Sucesión, Correspondencia y Oposición. Estas variables propuestas desde el estudio de la EC, operativizan la creatividad para trabajar una gramática corporal sin estereotipos. Esta gramática que permite comunicar características y mensajes personales, tiene también raíces comunes para poder encontrar un modo de comunicación con el otro. De esta forma cuestiones centrales como son el proceso empático, pueden estudiarse investigando variables dentro de la metodología de la EC, como por ejemplo las dinámicas de pasaje de movimiento con la idea de realizar un diálogo corporal empleando el conmovimiento y el contramovimiento (Schinca, 2010).

Incluir la EC en tan diversos ámbitos tiene como fundamento la relación que se ha establecido en las últimas décadas entre el conocimiento del cuerpo en movimiento y otros conceptos relevantes como la imaginación, la comunicación, el desarrollo personal y el teatro educativo.

Aunque el término Expresión Corporal abarque diferentes ámbitos e impregne a todas las artes del movimiento, también posee una entidad propia. La esencia que constituye esta disciplina es la búsqueda (a través del conocimiento, control y dominio del cuerpo) de unas formas de movimiento únicas. La Expresión Corporal como campo de conocimiento de específicas particularidades, tiene el valor de servir como herramienta para encontrar un lenguaje personal con el que poder comunicar y expresar, independientemente del dominio verbal. A partir de estos argumentos, la Expresión Corporal se puede considerar como disciplina independiente de otras artes del movimiento, con funcionalidad suficiente para ser aplicada a otros contextos humanos de expresión y comunicación.

\section{CONCLUSIONES}

Dado el creciente interés de la ciencia hacia los ámbitos artísticos y los estudios interdisciplinares de naturaleza creativa, se puede afirmar que las aportaciones de la formación actoral en EC, consiguen incorporar algunas bases para el trabajo en ámbitos de la comunicación humana, en principio alejados de lo artístico. Esta fusión puede desembocar en el desarrollo de herramientas concretas sobre el cuerpo en movimiento, para abordar problemas interactivos 
desde lo educativo y psicoterapéutico. Es el caso de la perspectiva psicológica psicoanálitica, donde la relación directa entre el lenguaje del cuerpo y la personalidad es claramente reconocida y estudiada. Cercanos a este paradigma, otros autores consideran el lenguaje corporal como una forma más de convivencia con uno mismo y con los demás. En esta línea, el trabajo del movimiento creativo con un fin terapéutico dispone de gran material de estudios e investigaciones (Espenak, 1989; Reca, 2017). Muchas veces la relación de la EC con la danza en el ámbito terapéutico, como disciplinas con objetivos comunes, se entremezclan para servir de vehículo que llevan a la rehabilitación y desarrollo de necesidades educativas especiales.

Indesligable del abordaje terapéutico de la EC, se encuentra también la aplicación de los principios e investigación corporal al ámbito social. En este punto destaca el trabajo de Paloma Santiago (2004), quien define la comunicación humana como comunicación corporal. El ser humano, en cuanto ser vivo, necesita comunicarse, concibiendo nuestro cuerpo como medio privilegiado de expresión. Respecto al aprendizaje de la EC, esta autora valora el trabajo intenso que permite el descubrimiento del movimiento propio, el gesto genuino.

El concepto de autoexpresión, equiparable con el termino Expresión Corporal, está en consonancia con la idea que el método Schinca (2010) desarrolla y trabaja en ámbitos educativos y artísticos. Este método observa en el estudio y la práctica de la EC, una forma de encontrar la expresión personal que emana de la conciencia corporal. Esto significa adquirir conciencia del propio cuerpo, con sus movimientos, emociones y modos de reaccionar. Tomar conciencia equivale, en gran medida, a adquirir el propio conocimiento y posibilidad corporal. Es en la función social e incluso terapéutica, donde la teleología comunicativa como fin último conecta el ámbito educativo, terapéutico y dramático. Se puede afirmar que el estudio y práctica de la EC establece canales de comunicación desde la unidad más básica: el movimiento. Esta idea ha sido mostrada en algunos trabajos desde la psicología del desarrollo, indagando sobre el factor comunicativo preverbal del periodo sensorio-motor del infante en sus primeros dos años de vida (Stern, 2010).

Es por esto que es necesario investigar los factores de movimiento implicados en diferentes situaciones de comunicación social. Adentrarse en las variables que permitan una exploración estructurada del campo de la Expresión Corporal, otorgará la estabilidad a una disciplina históricamente difusa y con límites desdibujados, con una importancia infravalorada. Todo lo anterior, junto con un adecuado y exhaustivo estudio de los factores de movimiento usados en el arte dramático, pueden ser perfectamente transferibles a otros ámbitos comunicativos y contextos de expresión cotidianos. Entender y manejar de manera consciente el lenguaje de movimiento, permitirá avanzar y catalizar procesos educativos y sociales, así como introducir claves relevantes en el estudio de los nuevos retos comunicativos.

\section{REFERENCIAS}

Abe, N. (2017). Towards a sociology of movement: Application of Kinetography Laban to the study of collective phenomena in the Paris subway. Social Science Information, 56, (2), 174-197. https://doi.org/10.1177/0539018417694775. 
Angotti, V. L., \& Herr, J. L. (1974). Etienne Decroux and the Advent of Modern Mime. Theatre Survey, 15(1), 1-17. https://doi.org/10.1017/S0040557400006141.

Artaud, A. (1986). El teatro y su doble. Barcelona: Edhasa.

Aslan, O. (1979). El actor en el siglo XX. Evolución de la técnica. Problema ético. Barcelona: Gustavo Gili.

Barba, E. (2003). Grandfathers, Orphans, and the Family Saga of European Theatre. New Theatre Quarterly, 19(2), 108-117. https://doi.org/ 10.1017/S0266464X03000034.

Barba, E. \& Savarese, N. (1988). Anatomía del actor. Diccionario de antropología teatral. México: Grupo Editorial Gaceta/International School Theatre Anthropology.

Bode, R. (2014). Rhythm and its Importance for Education. Body and Society, 20, 51-74. https://doi.org/10.1177/1357034X14546670.

Cohen, R. (2013). Acting Power. London and New York: Routledge. Taylor \& Francis Group.

Davis, F. (1998). La comunicación no verbal. Madrid: Alianza Editorial.

Decroux, È. (2000). Palabras sobre el mimo. México: El Milagro.

Doat, J. (1976). La expresión corporal en el comediante. Buenos Aires: Eudeba.

Ekman, P. \& Friesen, W. V. (1969). The repertoire of nonverbal behavior: categories, origins, usage and coding. Semiotica, 1, 49-98. https://doi.org/10.1515/semi.1969.1.1.49.

Espenak, L. (1989). Movement Diagnosis Tests and the Inherent Laws Governing Their Use in Treatment: An Aid in Detecting the Lifestyle. American Journal of Dance Therapy, 11 (2), 7783. https://doi.org/10.1007/BF00843772.

Ferrari, H. (2015). El enfoque de la enseñanza del movimiento según el método Schinca. Acotaciones, $\quad 35, \quad 115$ 149.http://www.resad.es/Acotaciones/index.php/ACT/article/view/40/100.

Finter, H. \& Griffin, M. (1997). Antonin Artaud and the Impossible Theatre: The Legacy of the Theatre of Cruelty. The Drama Review, 41 (4), 15-40. https://doi.org/10.2307/1146659.

García, I., Pérez, R. \& Calvo, A. (2013). Expresión corporal. Una práctica de intervención que permite encontrar un lenguaje propio mediante el estudio y la profundización del empleo del cuerpo. Retos, 23, 19-22. https://dialnet.unirioja.es/servlet/articulo?codigo=4135190.

Grotowski, J., Wiewiorowski T. K. \& Morris, K. (1967). Towards the Poor Theatre. The Tulane Drama Review, 11 (3), 60-65. https://www.jstor.org/stable/1125118.

Knapp, M.L., Wiemann, J.M. \& Daly, J. A. (1978). Nonverbal communication: issues and appraisal. Human Communication Research, 4 (3), 271-280. https://doi.org/10.1111/j.14682958.1978.tb00616.x 
Kotz, S. A., Ravignani, A., \& Fitch, W. T. (2018). The Evolution of Rhythm Processing. Trends in Cognitive Sciences, 22(10), 896-910. https://doi.org/10.1016/i.tics.2018.08.002

Laban, R. (1960). The mastery of movement. (2ªed.: London: Macdonald \& Evans, 1980).

Laster, D. (2012). Embodied memory: Body-memory in the performance research of Jerzy Grotowski. New Theatre Quarterly, 28(3), 211-229. https://doi.org/10.1017/S0266464X12000413.

Lecoq, J. (2003). El cuerpo poético. Barcelona: Alba Editorial.

Lust, A. (1971). Étienne decroux and the French school of mime. Journal Quarterly Journal of Speech, 57 (3), 291-297. https://doi.org/10.1080/00335637109383071.

Odom, S. L. (2005). Delsartean Traces in Dalcroze Eurhythmics. Mime Journal, 23, 136-151. https://doi.org/10.5642/mimejournal.20052301.09.

Oida, Y. (1993). From an Actor Adrift. Asian Theatre Journal, 10 (2), 202-212. https://doi.org/10.2307/1124180.

Reca, M. (2017). Reconstructing the World of Survivors of Torture for Political Reasons through Dance/Movement Therapy. The Oxford Handbook of Dance and Wellbeing, online publication. https://doi.org/10.1093/oxfordhb/9780199949298.013.35.

Rothe, K. (2012). Economy of human movement: Performances of economic knowledge. Performance Research, 17(6), 32-39. https://doi.org/10.1080/13528165.2013.775756.

Ruiz Osante, B. (2008). El arte del actor en el siglo XX. Un recorrido teórico práctico por las vanguardias (Teoría y Práctica). Bilbao: Artezblai.

Sainsbury, P. (1964). Muscle responses: Muscle tension and expressive movement. Journal of Psychosomatic Research, 8 (3), 179-185. https://doi.org/10.1016/0022-3999(64)90038-8.

Santiago, P. (2004). Expresión Corporal y Comunicación: Teoría y Práctica de un Programa. Salamanca: Amarú.

Schinca, M. (2010). Expresión Corporal: Técnica y Expresión de Movimiento. Madrid: Wolters Kluwer.

Seitz, J. A. (2005). Dalcroze, the body, movement and musicality. Psychology of Music, 33(4), 419435. https://doi.org/10.1177/0305735605056155.

Stern, D. (2010). The issue of vitality. Nordic Journal of Music Therapy,19 (2), 88-102. https://doi.org/10.1080/08098131.2010.497634.

Watson, I. (2013). The Odin actor: Embodied technology, memory, and the corporeal archive. Theatre, Dance and Performance Training, 4(3), 399-411. https://doi.org/10.1080/19443927.2013.824500. 
Whitehead, C. (2017). An explanation and analysis of one principle of Meyerhold's Biomechanics tormos. Theatre, Dance and Performance Training, 8(1), 89-102. https://doi.org/10.1080/19443927.2016.1214890. 